\title{
A VIRTUAL TESTBED FOR OPTIMIZING THE PERFORMANCE OF A NEW TYPE OF ACCELERATORS
}

\section{Maria Mingazova ${ }^{1, a}$, Alexander Degtyarev ${ }^{1}$, Alexander Bogdanov ${ }^{1}$}

${ }^{I}$ Saint Petersburg State University, 7-9 Universitetskaya emb., Saint Petersburg, 199034, Russia

E-mail: ${ }^{a}$ st048950@ student.spbu.ru

Simulation of the beam dynamics is an important stage in the design and launch of a charged particle accelerator. To solve this problem, many methods and software packages have been developed. Standard approaches to modeling accelerators require the use of not only ready-made libraries but exact theory and clearly formalized mathematical models. However, now such accelerator systems are emerging, where they plan to study phenomena, the physics of which is not completely clear. Therefore, a new type of accelerator requires a different approach to beam modeling. This article describes an approach to creating a virtual accelerator that will allow simulating particle physics, taking into account the peculiarities of the latest accelerators and the problem of the lack of an accurate theory for various phenomena planned to be detected at the accelerator.

Keywords: virtual testbed, GPGPU, modeling, beam accelerator

Maria Mingazova, Alexander Degtyarev, Alexander Bogdanov

Copyright $(C 2021$ for this paper by its authors. Use permitted under Creative Commons License Attribution 4.0 International (CC BY 4.0). 


\section{Introduction}

Particle physics modeling, reconstruction, and analysis software is an integral part of every high-energy physics experiment. One of the most important results of simulations is the creation of effective scenarios for maintaining the working process of such technological complexes as particle accelerators.

The development of such scenarios includes two items: selection of mathematical models and development the software for control systems. The first component is required for a preliminary study of various phenomena occurring in the accelerator facility. The simulation results are not only events expected to be observed at the accelerator but also sets of control parameters. The second component, software, is responsible for the implementation of operating mode scenarios in real installations based on the received data. The complexity of particle modeling lies in the need to apply multidimensional models with various mathematical formalizations, while sometimes the physics of processes may not be fully understood. The computational complexity of simulating and processing large amounts of data is driving the adoption of distributed computing systems such as supercomputers and cloud solutions.

For efficient control of large-scale complexes, it is advisable to use control systems based on virtual accelerator models. By combining simulation and analysis in a single software package, you can achieve significant savings in operating costs and investments in increasing capacity in order to maintain the speed of obtaining the result of experiments, while constantly increasing the data flow. As a result, we get a Virtual Testbed - a software environment that supports the design, virtual prototyping, data processing, and test.

\section{Virtual testbed}

The concept of a virtual testbed, namely the creation of a problem-oriented environment for fully functional modeling of the phenomenon under study or the behavior of a complex technical object, has now acquired a complete form. The development of this concept contributed to the development of complex mathematical models suitable for a full-fledged computational experiment and the improvement of a computer platform capable of providing the expected results.

The traditional type of virtual testbed is a computing environment for simulating complex processes, the physics of which is fully known. In this case, it is possible to carry out a complete simulation of the investigated phenomena. Such a type of virtual testbed is described in [1] where authors examine its concept and applications. However, there is another type of "virtual testing ground", when versatile modeling allows one to approach the understanding of phenomena, the physics of which is not yet fully clear and which require various experiments to understand the essence of the phenomena. For this, it is necessary to create experimental installations, on which new results are expected to be obtained. There is another option, the solution of the inverse problem, which may be mathematically incorrect and requires a large number of direct simulations for optimization.

Its key idea is to simulate particle physics using appropriate software packages (SixTrack [2], COZY Infinity [3], MAD [4], and others) with the ability to analyze, compare and optimize the results. The architecture of the virtual testbed allows you to organize sequential execution of tasks, in which the results of each stage of calculations based on the selected software package will be used as initial data for the next stage of calculations. For a software implementation, it is proposed to use a serviceoriented architecture - a modular approach based on providing remote use of distributed, easily replaceable components with standardized interfaces. This architecture allows the use of grid and cloud technologies, and also provides remote access to computing resources.

At the software level, a modular approach is also applicable. A virtual accelerator can be viewed as a set of programs and services that provide the execution of a computational process for simulating particle physics in accelerators using distributed computing power. The user interface of a virtual testbed should not only provide solutions on simulation models, but also provide the ability to 
identify the system, check the results, and optimize model configurations. The high-level layout of the virtual accelerator is summarized in Figure 1.

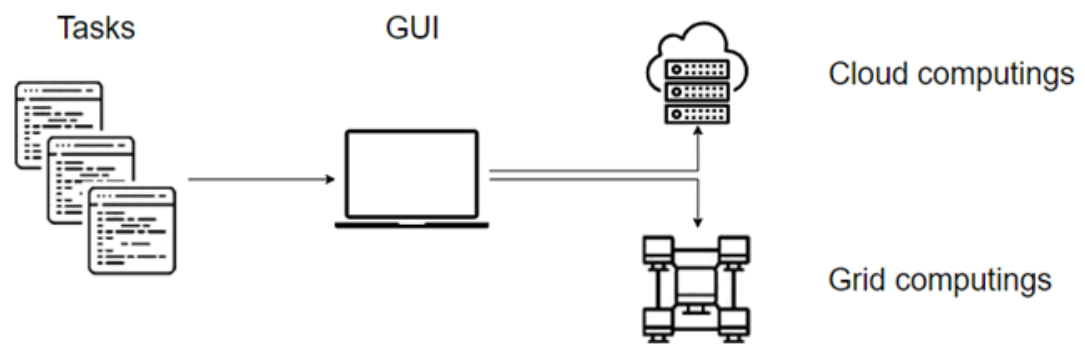

Figure 1. High-level diagram of a virtual accelerator environment

\section{Virtual testbed for new type of accelerators}

The key purpose of the virtual accelerator is to carry out computational experiments for particle physics simulations using appropriate software packages. The possibility of comparing the results (in the case of obtaining a solution to the same problem in different ways) and the ability to organize the flow of tasks (when solutions and the received data of the package are used as input data for subsequent calculations in the next package) are also an important function of the virtual testbed. Thus, the model can be considered as an integral part of the data processing system and monitoring data as input data for modeling. This will allow you to make more informed design decisions when developing the system. A more detailed description of the use of a standard virtual accelerator to simulate the launch of particles is described [5] and [6].

Despite the described advantages of using standard VTBs for analyzing and constructing accelerator control systems, this approach for a new type of accelerator (for example, NICA) no longer meets all the requirements for a virtual accelerator. The NICA project differs from the rest of the accelerators in energy and physics, which has not yet been fully explored. Therefore, it is proposed to solve the problem of finding effective control scenarios by carrying out numerous direct simulations. By varying the system configuration values, the result is a multidimensional set of suitable parameters.

In addition to finding the possible values of the system parameters by brute force, we can adjust the configurations using the operating data of the reproach station obtained from the monitoring tools. In this regard the following problems arise:

- verification of congruence of the input data for the simulations with the real parameters;

— verification that the calculations are performed correctly;

— verification that there are no differences in the behavior of the real system and the model.

For the new accelerators, the listed problems can be solved by generating hypotheses of the type of input data, system parameters, and the approaches of processing these data by modeling. Monitoring data from the accelerator operation can form the basis for formulating these hypotheses. To process the calculation results, the analysis of the temporal distribution of events generated during the processing of the input data is applied, and then these distributions are compared with the real system monitoring results.

Further, it is necessary to use optimization methods to find local or global extrema that give optimal values for scenarios of accelerator operation modes. The need for numerous runs of direct simulations leads to the fact that software optimization problems are an important part of computational experiments. In this case, parallel computing technologies can be effectively used. Each of the configurations can be performed independently of the other, and all of them form together parametric configurations of a given region of the accelerator. 
Thus, the virtual testbed is a distributed system for the design, modeling, processing, and visualization of data with unified access to all services. The user chooses the type of experiment, packages used, initial data, methods of implementation of calculations. The testbed assumes the coordination of data between packages, and also builds a pipeline of various stages of modeling.

Let us consider the proposed approach to the problem of creating scenarios for the effective operation of detectors. This problem is relevant for new accelerators that have been put into operation but are not tuned to the optimal operating mode. The development of such scenarios makes it possible to synchronize the operation of accelerators and event detection systems and reduce downtime. This task requires calculations for the entire chain of accelerators. Let us describe the steps that the virtual accelerator will take to build a computational experiment at one of the stages of particle acceleration.

To reconstruct particles in detectors, it is necessary to start with a detailed description of the geometry and properties of the chain of accelerators and detectors, and also indicate the parameters of the particle beam (Fig. 2, step 1). After entering the initial data, the user indicates the stages of the experiment for each of the accelerators in the system: event generation, event reconstruction, events visualization. Event generators are used to obtain particle distributions that are close enough to experimental ones. The tasks of reconstructing a charged track in experimental high-energy physics are pattern recognition (i.e., track search) and track fitting.

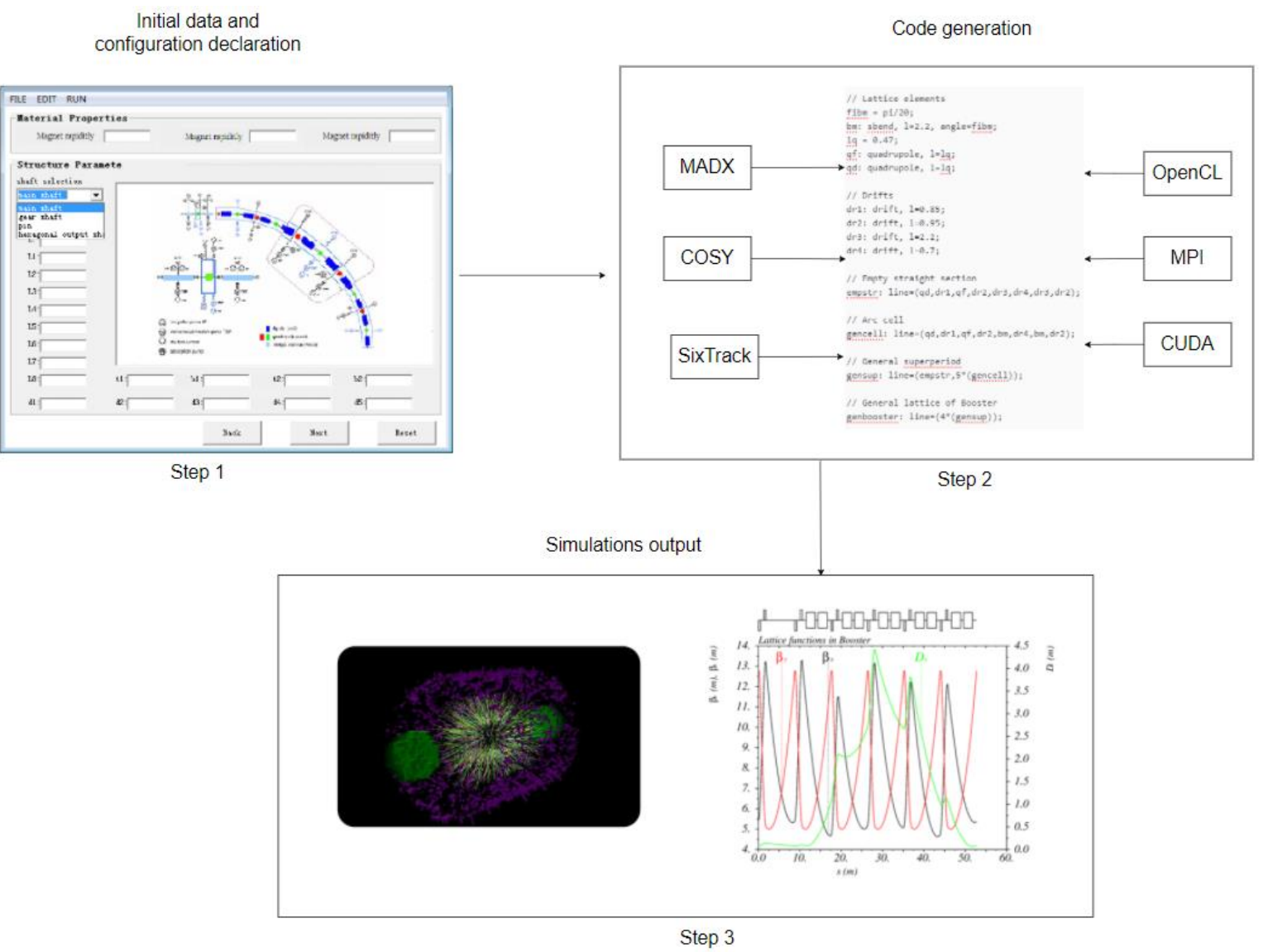

Figure 2. Schematic view of the Virtual Accelerator environment

For each stage, the user can select suitable packages, models, and numerical calculation methods (Fig. 2, step 2). The virtual accelerator will generate code to perform the modeling experiments. Grid and cloud technologies will allow running the same simulation scheme with different initial data at the same time, thereby parallelizing computations. After carrying out experiments on the virtual accelerator, it is necessary to process the results (Fig. 2, step 3) to obtain the optimal system configurations.

The main function of the proposed Virtual testbed is to provide a tool with high-level 
architecture for constructing experiments on modeling a particle beam in an accelerator. The advantage of such an environment is that the implementation details are hidden, the user interacts with the simulator only through the client layer. The user interface presents the state of the task execution, the measurements, and visualizes the results.

It should be noted that the Virtual Accelerator, being only a simulation environment, is not related to real control systems [7]. Although, such systems integration is possible.

\section{References}

[1] Bogdanov, A.; Degtyarev, A.; Gankevich, I.; Khramushin, V.; Korkhov, V. Virtual Testbed: Concept and Applications. Lecture Notes in Computer Science, vol.12254, 2020, pp. 1-15

[2] De Maria, R., et al. 'SixTrack Version 5: Status and New Developments'. In Proceedings of IPAC 2019, 3200-3203. Melbourne, Australia: JACoW, 2019. DOI:10.18429/JACoW-IPAC2019WEPTS043.

[3] K. Makino, M. Berz, "COSY INFINITY Version 9". Nuclear Instruments and Methods A558 (2005) pp. 346-350.

[4] Grote, H., and F. Schmidt. "MAD-X-an upgrade from MAD8."Proceedings of the 2003 Particle Accelerator Conference, Portland, OR. 2003.

[5] Kulabukhova, N. Andrianov, S.N. Bogdanov, A. Degtyarev, A. Simulation of space charge dynamics in high intensive beams on hybrid systems. Lecture Notes in Computer Science, vol.9786, 2016, pp. 284-295

[6] Kulabukhova, N. Bogdanov, A. Degtyarev, A. Problem-solving environment for beam dynamics analysis in particle accelerators. Lecture Notes in Computer Science, vol. 10408, 2017, pp.473-482

[7] B. Dalesio. EPICS V4 Expands Support to Physics Application, Data Acquisition, and Data Analysis. ICALEPCS 2011 